LA MONGDROMIA DE PICARD-LEFSCHETZ EN EL ESMUDIO DE LAS SINGULARIDADES COMPLEJAS.

V. NAVARRO AZNAR

INTRODUCCION

Los puntos singulares si bien han estado siempre presentes en el desarrollo de la geometría, su estudio ha estado, hasta mu recientemente, fundamentalmente reducido a un único problema: cb́mo hacer para dejar de estudiarlos. En efecto, lo que se buscaba con más ahinco no era conocer $\mathrm{y}$ entender los puntos singulares, sino hacer todo lo posible por evitarlos. El motivo bien evidente de ello estaba en el punto de vista birracional de la geometría y en el escuso interés que por el tema tenian otras ramas de las matemáticas. Desde hace unos veinte años el panorama ha cambiado notablemente y la aparición del punto de vista birregular en geometría, el estudio de interacciones elementales en física-matenática, el descubrimiento de estructuras exóticas en geometría diferencial y de variedades no diferenciables en topología han sido algunas de las. motivaciones que impulsaron el estudio de las singularidades, de manera que en la actualidad, habiendo tomado entidad propie, son objeto de enorme interés.

Fn esta exposicsón intentaré mostrar una bella forma, a mi entender, de clasificax los purtos singulares aislados de hipersuperficie compleja que, basada en la nocion de tipo topológico, perrite clasificerlos mediante su número de Milnor y su forma de seifert, de manera que -singularidades con distinto número de Milnor son de distinto tipo topologico; 
-sólo hay un múmero finito de singularidades de distinto tipo tcpologico con igual número de Milnor; $y$ finalmente

-singularidades con igual número de rilnor son del mismo tipo topolbgico si sus formas de Sejfert son igrales.

Este resultado constituirá el eje central de la narración que desearía fuera tan agradable para el lector como agradable es parc mí este tena.

El texto que sigue es una versión sensiblemente. revisada del que hubiera servido de base para la conferencia que pronuncié el 6.4.78 en la Uriversidad de Barcelona dentro del Seminario Earcelona de Matemáticas.

\section{TIPO TOPOIOGICO}

Si consideramọ dos gérmenes de subconjunto anaIltico, $(x, x)$ y $\left(X^{\prime}, x^{\prime}\right)$, la noción más inmediata de equivalencia es la analftica, que se expresa en la

Definición: Dos gérmenes de subconjunto aralítico, $(x, x)$ y $\left(X^{\prime}, x^{\prime}\right)$, son analfticamente equivalentes si sus anillos loceles, $\theta_{X, x}$ y $\theta_{X, X}$, , son -isomorfos.

No obstante, se observa que esta definición origine un criterio excesivamente fino de equivalencia, de forma que singularidades "aparentemente" iguales son analí ticamert,e distintas, por ejemplo el germen $\left(x_{a}, 0\right)$, ac $\mathbb{C}$, de ecuación local $x^{4}+a x^{2} y^{2}+y^{4}=0$ en $\mathbb{C}^{2}$, que pare todo acd corresponde a la intersección de cuatro rectas en el origen analiticamente depende del parámetro a, de forma que si a $\neq a^{\prime}$ sería $\mathrm{z}_{\mathrm{a}} \neq \mathrm{x}_{\mathrm{a}^{\prime}}$, sin embargo parece natural intentar en una primera clasificación considerarlas como una única singularidad. De hecho la imagen que se pretende seguir es la correspondiente a las superficies de Riemann: primero clasificerlas topológicamente seglín el género, después ya 
se resolverá el problena de la equivalencia cunforme (problema de los modulos) para cada sénero.

Enterdida así la clestión la clasificación grosera que se busce puede obtenerse, en el caso de hipersuperficies, basándose en la sjguiente

Definición: Sean $(\pi, 0)$ y $\left(X^{\prime}, 0\right)$ dos gémenes de ripersuperficit de igual. dimesibn. Diremos que tieren riswo tipo topologico si exister representantes locales $(x, 0) \subset\left(\mathbb{c}^{n+1}, 0\right), \quad\left(x^{\prime}, 0\right) \subset\left(\mathbb{a}^{n+1}, 0\right)$ y wi germen de homeomorfismo $\left(\mathbb{C}^{n+1}, X, 0\right) \approx\left(\mathbb{C}^{n+1}, X^{\prime}, 0\right)$.

Alanque esta definjoion no requiere que las singularidades sean aisladas, en Io sucesivo nos ocuparemos exclusivamelite de este caso por ser los.resultados entonces mucho más fáciles y completos. Así pues, empezaré citando este criterio

Leme: Sea $(\bar{X}, 0)$ un germen de hipersuperficie en $\mathbb{C}^{n+1}$, sea $f=0$ une ecuación local de $(X, 0)$ y sea $f(f)=\left[D_{1} f, D_{2} f, \ldots, D_{n} f\right]$. Entonces $(X, O)$ presenta a 10 sumo una singularidad aislada en el origeri sii

$$
\operatorname{dim} \frac{\theta_{n+1}}{\$(f)} \text { es finita. }
$$

Como puede observarse fácilmente esta dimension no depende de la ecvación locki de $(x, 0)$ escogida y permite Ia:

Definicion: Se llama número de Milnor de $(X, 0), \mu(X)$, a la dimension sobre de

$$
\frac{\theta n+1}{y(f)}
$$

Con esta nomenclatura se tiene, de forma trivial: un germer $(X, O)$ es regular sii su numero de Milnor es cero, criterio éste que será de utilidad más adelante; y un germen $(\mathrm{X}, 0)$ presenta a 10 sumo una singularidad aislada en el origen sii su número de Milnor es finito.

Ejemplo: Procuraré er lo que sigue ir mostrando todos los conceptos que vaya introduciendo sobre un aso concreto, 
será éste el de la serie ce singularidades cuspidales, A que admiten como ecueción local $x_{0}^{m+1}+x_{1}^{2}+\ldots+x_{n}^{2}=0$, parct éstas verios fáci]mente, apj.icanojo la definicjón, que su número de Milnor es m.

Acabanos de asncier a un gexmen coin sirgularidao aislada un número natural, ahora venos a asociarle un nudo er coditension äos que permite una buene representación. topolófica de la singuiaridad, es ésta

Teorema 3e estructura conica 1 (ver [33]): Sea $(x, 0)$ un germen de hipersuperfjcie er! $\mathbb{C}^{n+1}$ con singularidad aislada, $y$ sea $V$ un representante de $(X, 0)$ en un entomo abierto $U$ del origen. Entonces existe un $\varepsilon>0$ tal que:

i) $\mathrm{B}_{\varepsilon}=\left\{x \in \mathbb{C}^{n+1} ;\|x\| \leqslant \varepsilon\right\} \subset \mathrm{U}$

ii) $S_{\varepsilon}=\left\{x \varepsilon \mathbb{C}^{n+1} ;\|x\|=\varepsilon\right\}$ corta a $V$ en una subvariejäd (2n-1)-dimensional, $(n-2)$-conexa, $\mathrm{K}_{\varepsilon}$.

iii) el far $\left(B_{\varepsilon}, V \cap B_{\varepsilon}\right)$ es homeomorfo al conor $\left(S_{\varepsilon}, K_{\varepsilon}\right)$.

iv) si $\varepsilon>\varepsilon$ '>0 los pares $\left(S_{\varepsilon}, K_{\varepsilon}\right)$ y $\left(S_{\varepsilon}, K_{\varepsilon},\right)$ son difeotbpicos, a la clase de difeotopía de estos nudow se 11 inta el nudo de? germen $(\mathrm{x}, 0)$.

Así se tiene que dos gérmenes son del mismo tipo topológico sii sus nudos son equivalertes.

Ejemplo: Continuando con la serie $A_{\text {m }}$ vamos a considerar ahora sólo $n=\uparrow$, esto es singularidades de curves Flancs, pues precisamente fue con el estudio topológico de estas singularidades por Brauner [5], Burau [10], [11], y Kahler [23], cono se inicib e: método que acabamos de citar de los nudos. Por consiguiente las singularidades a considerar sor las de ecuación local $x^{m}+y^{2}=0$ en $\mathbb{t}^{2}$, pero ahora no es dificil comprober que proyectando estéreograficamente la $\mathrm{S}^{3}$ sobre $\mathrm{R}^{3}$, el enlazado $\left(\mathrm{S}^{3}, \mathrm{~K}\right)$ es precisamente, si m es impar, el bien conocido nudo tórico $(2, \mathrm{~m})$, y si m es par, dos circhrferencias sin nudos enlazadas con numero de enlace $\mathrm{m} / 2$ (ver fig. 1) 


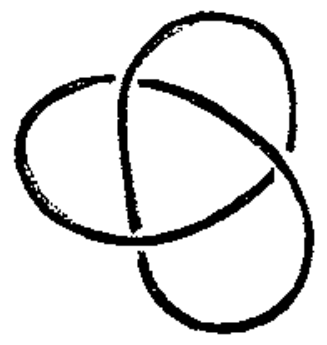

II $=3$

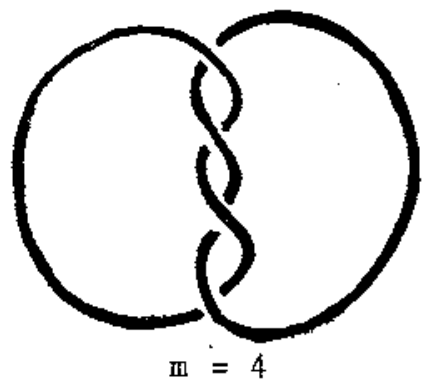

Figura 1

\section{IA EIBRACION DE MILNOR}

Er este apartado vamos a introducir una estructura sobre eI nudo $\left(S_{\varepsilon}, K\right)$ anterior, la fibracjón de Milnor, que desempeñ: un papel esercial en la clasificación de gérmenes basade en el tipo topológico.

Históricamente, la estmetura a le que re refiero se derivó dei nétodo de las secciones hiperplancs que s. Lefschetz [30] introdujo en su estudio de la topología de las variedades proyectivas. Empezaré explicando cómo se entiende ahora este rétodo, sin entrar en detalles.

Sea $Y$ una variedad compleja lisa de dimensión $n+1$ y $f: Y \rightarrow \mathbb{c}$ una función holomorfa, propia y con un núrero finito $a_{1}, \ldots, a_{r}$ de puntos críticos, no degenerados $(\mu=1)$, y con valores críticos distintos $b_{,}, \ldots, b_{r}$ respectivamente. Tomando un $\delta>0$ suficiertemente pequeño los discos $D_{j}=\left\{z \varepsilon ;\left|z-t_{j}\right| \leqslant \varepsilon\right\}, 1 \leqslant j \leqslant r$, serán disjuntos dos a dos, sea entonces $w_{j}(t)=b_{j}+\delta e^{2 \pi i t}, 0 \leqslant t \leqslant 1$, y sea $u_{j}$ un camino $C^{\infty}$ en $\mathbb{C} \sim\left\{b_{1}, \ldots, b_{r}\right\}$ uniendo un punto base $\mathrm{z}_{0} \varepsilon-\left\{b_{1}, \ldots, \mathrm{b}_{r}\right\}$ con $b_{j}+\delta$. Ilamaremos entonces $a$

$$
v_{j}=u_{j}^{-1} w_{j} u_{j}
$$

un camino elepental en torno $a b_{j}, y$ a su clase de homotopía en $\pi_{1}\left(c-\left(b_{1}, \ldots, b_{r}\right), z_{0}\right)$ la notaremos $f_{j}$.

con estas hipótesis y notaciones e? resultado de Iefschetz es éste 
Teorema z (ver $[30],[15],[27\rfloor):$

i) $f_{b}: Y-f^{-1}\left(\left\{, \ldots, b_{n}\right) \rightarrow \mathbb{C}-\left\{b_{1}, \ldots, b_{r}\right\}\right.$ es un fibrado $C^{\infty}$ localmente trivial, $y$ por tanto todes sus fibras sor difeomorfas.

ii) si $F=f^{-1}\left(z_{0}\right)$, la aplicación

$$
\mathrm{H}_{i}(\mathrm{~F}, \mathrm{z}) \rightarrow \mathrm{H}_{i}(\mathrm{Y}, z)
$$

es biyectiva si i<n, y exhaustive si $i=n$.

iii) existe para cada clese $\beta_{j}, 1 \leqslant i \leqslant r$, un cicio $s_{j} \varepsilon H_{n}(F, Z)$, de manera que

$$
N u ́ c\left(H_{n}(F, Z) \rightarrow H_{n}(Y, Z)\right)=\left[s_{1}, \ldots, s_{r}\right]
$$

a este núclec se le llama el grupo de ciclos evanescentes.

En el apartado 3 completaremos estos resultados viendo como los ciclos evanescentes $s_{j}$ permiten el cálculo de la monodromía de Picard-Lefschetz.

Volvamos ahora a considerar un germen $(x, 0)$ de hipersuperficie en $\mathbb{f}^{\mathrm{n}+1}$ con singularidad aislada, si $f=0$ es una ecuación local de $(X, 0)$ definida en un entorno abierto $U$ de 0 en $\mathbb{C}^{n+1}$, la aplicación $f: U \rightarrow \mathbb{C}$ presentia un único punto crítico en 0 , escogiendo $J$ adecuadamente. Así tenemos una situación parecida a la anterior pero en la que, generalmente, ni f es propia ni el punto críticc es no degenerado. La construcción que en estie caso realiza Milnor es la siguiente: considerando el nudo $\left(S_{\varepsilon}, K\right)$ define

$$
\begin{aligned}
\varphi: S_{\varepsilon}-K & \rightarrow s^{\dagger} \\
x & \rightarrow f(x) /\|f(x)\|
\end{aligned}
$$

y entonces obtiene el siguiente resultado:

Teorema 3 (Milnor [33]):

i) Si $\varepsilon>0$ es suficientcmente pequeño, $\varphi$ es un fibrodo $\mathrm{C}^{\infty}$ localmente trivial. 
ii) $F=\varphi^{-1}(i)$ es el interion de una variedad diferencjable con borde, 2n-dimereional, y cuyo borde es precisemente $K$.

i.ij.) $\tilde{H}_{i}(F, z)=0$, si $i \neq n$.

iv) si $\mu$ es el número de Milnor de $(X, 0)$ entonces $\tilde{\mathrm{H}}_{\mathrm{n}}(\mathrm{F}, Z)$ es libre de rango $\mu$.

v) Si f' es otra ecuación local de $(X, 0)$ y si $\varepsilon$ '>0 es sufjecientemente pequeño pera cue $\varphi^{\prime}$ cumpla i), entonces los fibrados ' $\varphi$ y $\varphi$ ' son diferenciablemente equivalentes.

Al fibrado que define el teorema se le llame

fibración de Milnor del germen $(X, 0)$, y a su fibra tipica $F$, la fibra de Milnor del germer $(X, 0)$.

Ejemplo: Para la serie $A_{m}: x^{m+1}+y^{2}=0$ henos visto anterior merite que $\mu=m, y$ que $K$ era ei erlazado $(2, \pi)$, csí es fácil dar un buen modelo de la fibra de Milnor $F$, ver fig. 2:

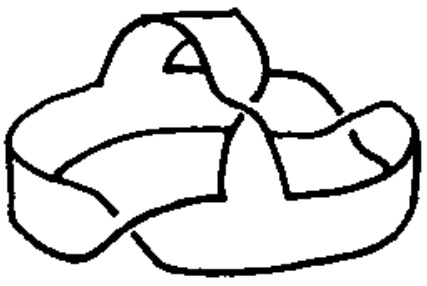

$\mathrm{m}=3$

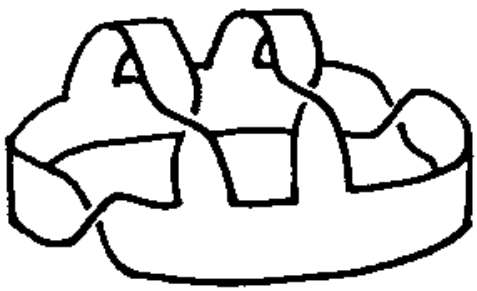

m $=4$

Figura 2

Existe otre construcción, también de Milnor, que define una fibración equivalente a la anterior, y a la cual me referiré en el apartado 4, es ésta: sea $B_{\varepsilon}=\left\{z \varepsilon \mathbb{C}^{n+1} ;\|z\|<\varepsilon\right\}, \quad T_{\eta}=\{z \varepsilon \mathfrak{C},\|z\|<\eta\}$, $\mathrm{T}_{\eta}^{*}=\mathrm{T}_{\eta}-\{0\}, y$ noteros $\mathrm{X}_{\varepsilon, \eta}^{*}=\mathrm{B}_{\varepsilon} \cap \mathrm{f}^{-1}\left(\mathrm{~T}_{\eta}^{*}\right)$, entonces si $\varepsilon$, $\eta$ son convenientemente escogidos,

$$
f: X_{\varepsilon, \eta}^{*} \rightarrow T_{\eta}^{*}
$$


es un firjiso $C^{\infty}$ Joce Imerte trivial, e isomorfo al del tecrem. $\therefore$ : ver fig. 3:

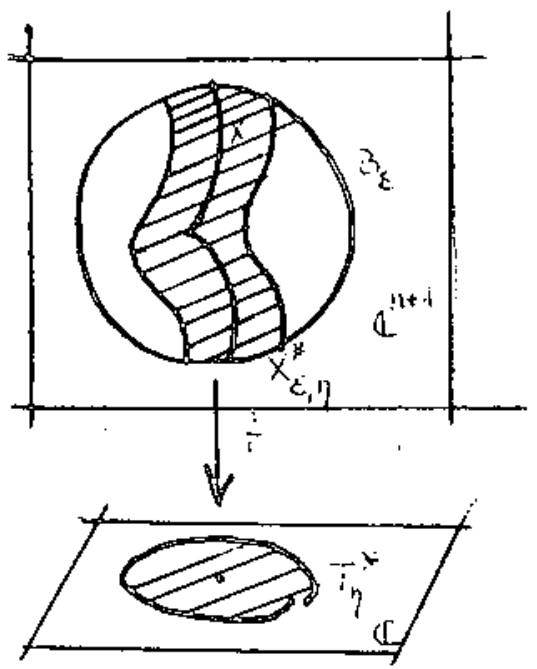

Figura 3

ia importancia de la fioración de Nilnor con respecto a la clasifickción cue pretento exponer, está en que a partir de elle se obtiene este

Teorema 4 (ver $[1],[2 \&]): \operatorname{Sean}(X, 0) y(X, 0)$ témeries de hipersuperficie en $\mathbb{0}^{n+1}$ del mismo tipo topolbgico, entonces

i) $(X, 0)$ es regular sii $(X, 0)$ es regular, i.e. $\mu(\bar{X})=0$ sij. $\mu\left(X^{\prime}\right)=0$;

i.i) $(X, 0)$ posee wi punto singuier aislado en ei oxigei sii $\left(X^{\prime}, 0\right)$. Io posee; $y$ en este caso

iii) $\mu(X)=\mu\left(X^{\prime}\right)$.

Con este resultado vemos que el. múmero de Milnor es un buer indieador del tipo topológico de la singularidad; que también es un cómodo indicador resulta del siguiente teorema, conjeliurado por R. Thom, y que justifica, en parte, la introducción misma del concepto tipo torológico. 
Teorema 5 (Fukuda [16]): Sólo hay ur múmelo finto de bipos topológicos distintos de gémeres de hipersuperficie con singular-idad aislada, que posear el mismo nímero de Milror.

Antes le dejar este apartajo quiero comenter qué otre exigencia aebería cumplir la ciasificación basada en el tipo topológico parr een una muy buena clasificación. Aurque, en el epertaōo 5 haré otras precisiones, me limito ahora $z$ ie multiplicidad, en efecto, hasta ei viorerto no hatía habilado de la multjilicidad del gertier $(x, 0)$, $n(x)$, aún cuando está claro que es un importante invariante analítico, recuérdese que $\mathrm{m}(X)=1$ sii $(X, 0)$ es regular. Ira razón de este olvido está en que la multidlicidad no es un buen indicador, ni siquiera anelítico, basta pensar er la serie $A_{m}: x^{\Pi+1}+y^{2}=0$, toda ella de multiplicidad 2. Ahora. bien, lo que sí sería muy de desear es cue la clasizícación topológica que estamos propugrando fues'a confatible con le maltiplicidad, esto es, desearíanos tener ure respuesta positiva a la

Pregunta (Zariski, $[46])$ : ji $(X, 0)$ y $\left(X^{\prime}, 0\right)$ son gérrenes de hìpersuperficie del mismo tipo topológico ces $\mathbb{H}(X)=m\left(X^{\prime}\right)$ ?

En el apartado 5 se dará una tabla soine la cual puede verse que diche pregunta es cierta si $1 \leqslant \leqslant 10$. Pero una forme de atcque general a este prublem, parece que debe estar basada en la fibrsción de Milnor de $(x, 0)$, en especial seria muy interesante une respuesta positiva a la

Pregunta: Si $(X, 0)$ y $\left(X^{\prime}, 0\right)$ son zérmenes de hipersuperficie del mistio tipo topológico z son sus fibraciones de vilinor homeombricanente equivalentes?

Efectivamente esto es asi en todos los casos que conozce, ver en especial [24] y [29], y resultaría por los resultados de [24] de esta última

Pregunta: Sea $(x, 0)$ un germen de hipersuperficie ccn singularidad aislada iexjste un germen de hipersuperficie $\left(X^{\prime}, 0\right)$ del raisuro tipo topológico que $(X, 0)$, y que admita una ecuación locel $f=0$, con $f$ un polinomio real? 
Corro ya he mencionado, estas dos últirias pregunthas admiten respuesta afirmativa si $n=1$ (caso de curvas Fianas), en todas Ias fomas normeles de [3], y en todes Ias familias a $\mu$ constante que sean quasi-homogénecs.

3. MONODROMIA

Al disponer ahosa de la fibración de Milnor asociada al germen $(x, 0)$, podemos obtener una información todavía mayor si consideramos la monodromía de dicha fibracion. Recordemos que dade una fibracion $\varphi: E \rightarrow B$, se pueden levantar los caminos trazados sobre la base $B$ en caminos sobre el espacio total $\mathrm{E}$, de forma que a ceminos homótopos en $B$ corresponden caminos homótopos en $E$, en particular si consideramos caminos cerrados en $B$ con punto base $b$, obtendrenos homeomorfismos de la fibra $F_{b}$, y ccmo a una clase de homotopía en $\pi_{j}(B, b)$ corresponde una clase de homeomorfismos homotopos er $F_{b}$, resulta en definitiva un morfismo

$$
\pi_{1}(\mathrm{~B}, \mathrm{~b}) \rightarrow \operatorname{Aut}\left(\mathrm{H}_{*}\left(\mathrm{E}_{b}, \mathrm{Z}\right)\right)
$$

a esta aplicación se le Ilana la monodromía de la fibración, y a su imagen se le Ilama el gmuno de monodrouría de la fibración.

En el caso de la fibración de tefscheta, esta monodrowía, por tener su origen en Ficard [37] recibe el nombre de Monodromía de Picard-Lefschetz, y está explicitamente determinada en el siguiente Teorema 6 (Fórmulas de Picard-Lefschetz, ver [37], [30], [15], [27]): Con las hipótesis y notaciones del teorema 2, la monodromia de la fibración $f$ hace corresponder a cada clase $\beta_{j} \varepsilon \pi_{f}\left(\mathbb{C}-\left\{b_{1}, \ldots, b_{n}\right\}\right)$ el automorfismo $h_{j *}$ de $H_{*}(F, z)$ definido por

$$
\begin{aligned}
& h_{j, q}=i d \text { si } q \neq n \\
& h_{j, n}(y)=y-(-1)^{\frac{n(n-1)}{2}}<y, s_{j}>s_{j}, \text { para todo }
\end{aligned}
$$


En el caso de la fibración de Milnor que nos ocupa comó el espacio base de la fibración es la $S^{1}$ y como la homología de 13 fibra es trivial salvo en dimenzín $n$, esta monodromía queda deterrinada si danos el autonortismo $h_{*}$ de $H_{n}\left(F_{b}, z\right)$ que corresponde al generador "positivo" de $\pi_{1}\left(S^{1}, b\right)$, a este automorfismo $h_{*}$ se le llana el operador. de morodromia, o bieri la monodromía aleebrsica, de la fibracjor de Milnor.

En este punto merece resaltarse un resultado análogo local al que desempeña un importante pepel en Ia denostración de Deligne de las conjetures de Weil.

Teorema de monodromía 7 (ver [9]): El operaảor de monourcmía es quesi-unipotente, i.e. existe un i>0 tal que $\left(n_{*}^{i}-I\right)^{n+1}=0$.

En particular, si llamamos polinomis de monodromía, $\Delta(t)$, al polinomio característico de $h_{*}$ se tiene: las raíces det polinomio de monodromía son raíces de la unidad. Ejemplo: Si quiero seguir ilustrando lo que estoy diciendo con el ejerplo $x_{0}^{\mathrm{m}}+\mathrm{x}_{1}^{2}+\ldots+\mathrm{x}_{n}^{2}=0$, deberé considerar la fibra de Milnor $F=\left\{x_{0}^{m}+\ldots+x_{n}^{2}=1\right\}$, en cuyo caso $h_{*}$ estará inducja por la monodromía geométrica

$h\left(x_{0}, \ldots, x_{n}\right)=\left(e^{\frac{2 n i}{m}} x_{0}, \ldots, e^{n i} x_{n}\right)$, pero para evitar excesivas comprobaciones, utilizars este teorema de Sebastiani-Thon! [38]:

Si $f(x, y)=f_{0}(x)+f_{1}(y)$ con $x \in \mathbb{E}^{n}, y \in \mathbb{C}^{n}, y$ si $f_{0}(x)$, resp. $f_{1}(y)$, presenta una singldaridad aislada en Oe $\mathbb{C}^{n}$, resp. Oe $\mathbb{C}^{\mathrm{a}}$, entonces $f(x, y)$ preserta una singularidad aislade en $(0,0) \varepsilon \mathbb{c}^{n_{x}} \mathbb{C}^{m}, \quad \tilde{H}_{n+m-1}(F)=\tilde{H}_{n-1}\left(F_{0}\right) \otimes \tilde{H}_{n-1}\left(F_{1}\right)$ y $h_{*}=h_{* 0} \otimes h_{* 1}$.

Con esto ya es muy fácil pues si $f\left(x_{0}, \ldots, x_{n}\right)=$ $=x_{0}^{m}+x_{1}^{2}+\ldots+x_{n}^{2}$ tendremos $f_{0}\left(x_{0}\right)=x_{0}, \ldots, f_{n}\left(x_{n}\right)=x_{n}^{2}$, así que bastará con encontrar la monodromía de $f_{c}$, pero en este caso la fibra está constituida por las raíces n-ésimas de la unidad, y la monodromáa algebraica en $H_{0}(F)$ es sencillamertie decalar uno, asi 


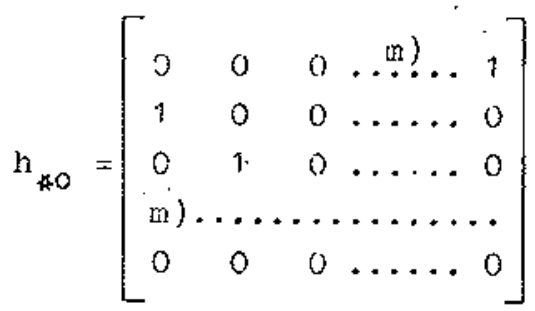

ro obstante, al estar interesados en $\tilde{\mathrm{H}}_{j}(F)$ debenos terer en cuenta que, si $\left(e_{1}, \ldots, e_{m}\right)$ es una base de $H_{0}(F)$, ura base de $\tilde{H}_{0}(F)$ es, por ejeriplo, $\left(e_{1}-e_{n i}, \ldots, e_{m-1}-e_{i n}\right)$ y er esta base

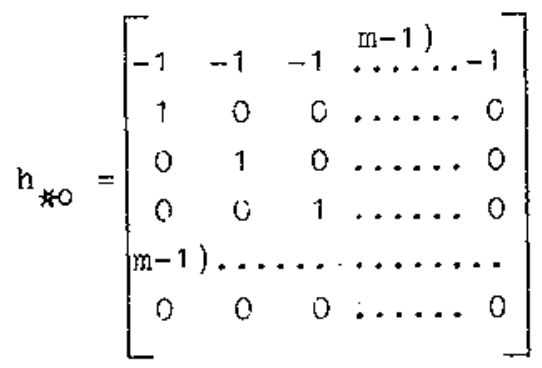

en definitiva pues

$$
n_{*}=n_{* 0} \otimes n_{* 1} \otimes \ldots \otimes n_{* n}=(-1)^{n_{n}} n_{* 0}
$$

Esta matriz nos permite calcular el polinomio de monodromía, resultando evidentenente

$$
\Delta(t)=\frac{t^{m}-1}{t-1}
$$

Vamos a ver, por últino, como se define la forma de Seifert a partir de la morodromía algebrajca. Pare ello deberé recordar la definición clásica de número de enlace: si $x, y$ son $n$-ciclos en $s^{2 n+1}, n \geqslant 0, y X$ es una $(n+1)$-cadena tal que $\partial \mathrm{x}=\mathrm{x}$ entonces el número de erlace de $\mathrm{x} \operatorname{con} \mathrm{y}$, $\mathrm{I}(\mathrm{x}, \mathrm{y})$, es el número de intersección de $\mathrm{X}$ e $\mathrm{y}$, así $l(x, y)=\langle X, y\rangle$. Con esto la forma de Seifert se define mediante: 


$$
\text { s: } \begin{aligned}
\tilde{\mathrm{H}}_{\mathrm{n}}(F) \times \tilde{\mathrm{H}}_{\mathrm{n}}(F) & \rightarrow \mathrm{Z} \\
(\mathrm{x}, \mathrm{y}) & \rightarrow I\left(\mathrm{~h}_{\pi} \mathrm{x}, \mathrm{y}\right)
\end{aligned}
$$

donde $h_{\pi}$ es ez isororfismo $\tilde{H}_{n}\left(F_{0}\right) \rightarrow \tilde{H}_{n}\left(F_{\pi}\right)$ inducidc por el canino $w:[0, \pi] \rightarrow s^{1}, w(t)=e^{i t}$.

Ia forma de seifert resulta ser no degenerada y su importancia redica eri $e$ ?

Teorema 8 (Levine [3], ver también [12]): Sean $(x, 0)$ y $(x, 0)$ dos gémenes de niversuperficie er: $\mathbb{C}^{n+1}$ con singularidad aislada. Si sus formas de seifert son equivalentes, entonces $(X, 0)$ y $(X, 0)$ son del misto tipo topológico. - Es importante hacer notar que la forma de seifert facilita información explícita sobre la topología del german; en efecto: en primer lugar muestra el número de Milnor, como es evidente, en segurdo luger, da la estructura multiplicajiva de $\tilde{H}_{n}(\boldsymbol{F})$ pues si i denota la forma le intersección en $\tilde{H}_{n}(p)$ se tiene (ve: $\left.: 12\right]$, [27])

$$
i=s+(-1)^{n} s^{t}
$$

y en tercer lugar, permite obtener la monodromia algebraica de la fibración de Milnor, pues tomando una base en $\tilde{H}_{n}($ F) resulta (ver [12], [27]) le igueldad motricia]

$$
H=(-1)^{n+1} S^{-1} S^{4}
$$

Ejenplo: Para las singularidaces de la serie $A_{n}$ se encuentra que su forma de Seifert admite como itetriz, (ven [12])

$$
S=(-1)^{\frac{n(n+1)}{2}}\left[\begin{array}{cccccc}
1 & 1 & 0 & \ldots & \ldots & 0 \\
0 & 1 & 1 & \ldots & \ldots & 0 \\
0 & 0 & 1 & \ldots & \ldots & 0 \\
\text { mil } & \ldots & \ldots & \ldots & \ldots & \ldots \\
0 & 0 & 0 & \ldots & \ldots & 1
\end{array}\right]
$$


Quiero exponer aurora una constricción que, eúl siende también local, pennitie una visión mucho más estereográfica de la singularidad, puess nientris se represerita en uno de sue planos la fibración de Milnor, en otros planos se representan escenas (fibraciones) no degeneradas de Picard-Lefschetz. Ia idea os "eumergir" la singularided dada en un espacio de dimension mayor, de tai forma que dicho espacio contenga "todas" lás singulariades "fróxinas" a la origina?. Precisar las anteriores palabras es introducir la noción de deformación semiuniversal. Definición: $S i\left(X_{0}, x_{0}\right)$ es un germen ce espacio analítico, una deformación plana, o simplemente deformacion, de $\left(\mathrm{x}_{0}, \mathrm{x}_{\mathrm{C}}\right)$ es un cuedrado cartesiano

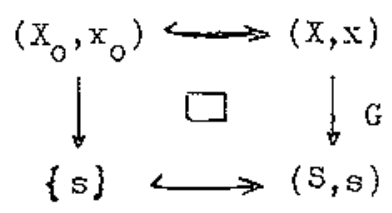

en el que $G$ es un morfismo pleno. Al espacio $(X, x)$ se le liame especio total, $y$ al $(S, s)$ espacio base, o espacio de paránetros, de 1 il deformeción.

Se definen de forma eviderte los morfismos entre ceformaciones de un germen, y las deformaciones por cimhio je base. Entonces, se dice de una deformación que es versal si todas las otras deformaciones de aquel germen se obticnen por canbto de base a partir de dicha deformacion versal, $y$ se dice de una deformación versal que es semi-universal s.. la dimensión de su espacio base es mínima.

El problenia clave a resolver es el de existencia de deformaciones versales de un germer dado, y el de caracterización de las semiuniversales. Este problema que en el caso general es muy difícil y sćlo ałmito solución hajo deterninadas hipótesis (ver [19], [44]), tjene en el caso que estamos tretando de hipersuperficies con singularidad aislada una respuesta simple: 
Tecrema 9 (ver $[\Delta 4])$ : Si $\left(\mathrm{X}_{0}: \mathrm{x}_{0}\right)$ es un gemen de hipersuperficie con singularidad aislada y de número de Milnor $\mu$, entonces existe una deformación senimiversal de $\left(x_{0}, x_{0}\right)$, siendo la dimension c del espacio base de ésta $\leqslant \mu$.

Pero más interesante todavía es que este defornación puede construirse explícitamente como sigue. Sea $f=0$ una ecuación local de $\left(\mathbb{X}_{0}, x_{0}\right)$ y sea $\left(e_{1}, \ldots, e_{r}\right)$ una base sobre $\mathbb{C}$ de $\frac{\theta_{X_{0}, x_{0}}}{[f, \mathcal{f}(f)]}$, entonces el espacio total $(X, x)$ de la deformación seniuniversal que buscamos es simplemerte el gemen en $x=\left(x_{0}, 0\right) \varepsilon \mathbb{t}^{n+1} x \mathbb{d}^{\top}$ del espacio analítico en $\mathbb{a}^{n+1} \times \mathbb{C}^{x}$ con ecuación local

$$
f(x)+e_{1} t_{1}+e_{2} t_{2}+\ldots+e_{\tau} t_{\tau}=0
$$

el espacio base $(S, S)$ es $\left(\mathbb{C}^{\top}, 0\right)$, y $G$ no es más que la restricción $\mathrm{a},(\bar{X}, \mathrm{x})$ de la proyección natural.

Si $(C, x)$ es el espacio crítico de $G, y(D, 0)$ su espacio discriminante, es decir la imagen analítica directa de $(\mathrm{C}, \mathrm{x})$ por $\mathrm{G}$, se tiene que, tomando representantes, $D \mathrm{da}$ los valores de los parámetros para los cuales la fibra es singular, y resuita el

Teorema 10 (Teissier [40], Gabrielov [18]): (c,x) es liso y $(D, 0)$ es un germen irreducible de hipersuperficie en $\mathbb{C}^{\mathrm{T}}$; de multiplicidad $m(D)=\mu$.

Más concretamente, si en la base $\left(e_{1}, \ldots, e_{\tau}\right)$ escogida anteriormente suponemos $e_{\tau}=1$, entonces $\left(t_{1}, \ldots, t_{\tau-1}\right)$ forman un sistema de parámetros para $D$, de manera que, tomando representantes, la restriccson a $D$ de la proyección $\varphi: \mathbb{C}^{\top} \rightarrow \mathbb{C}^{\top-1}$ es un recubrimiento analítico ramificado de orden $\mu$, cuyo lugar de ramificación $\Delta$ tiene dos componentes irreducibles: la cáustica, $\Delta_{1}$, sobre la cual están las fibras singulares con singularidad degenerada, $y$ el estrato de Maxwell, $\Delta_{2}$, sobre el. cual están las fibras con más de una singularidad. 
Ejemplo: Me Iimitaré a ilustrar gráficamerte le deformación seniuniversal pera $f=x^{3}$, en cuyo caso se tiene

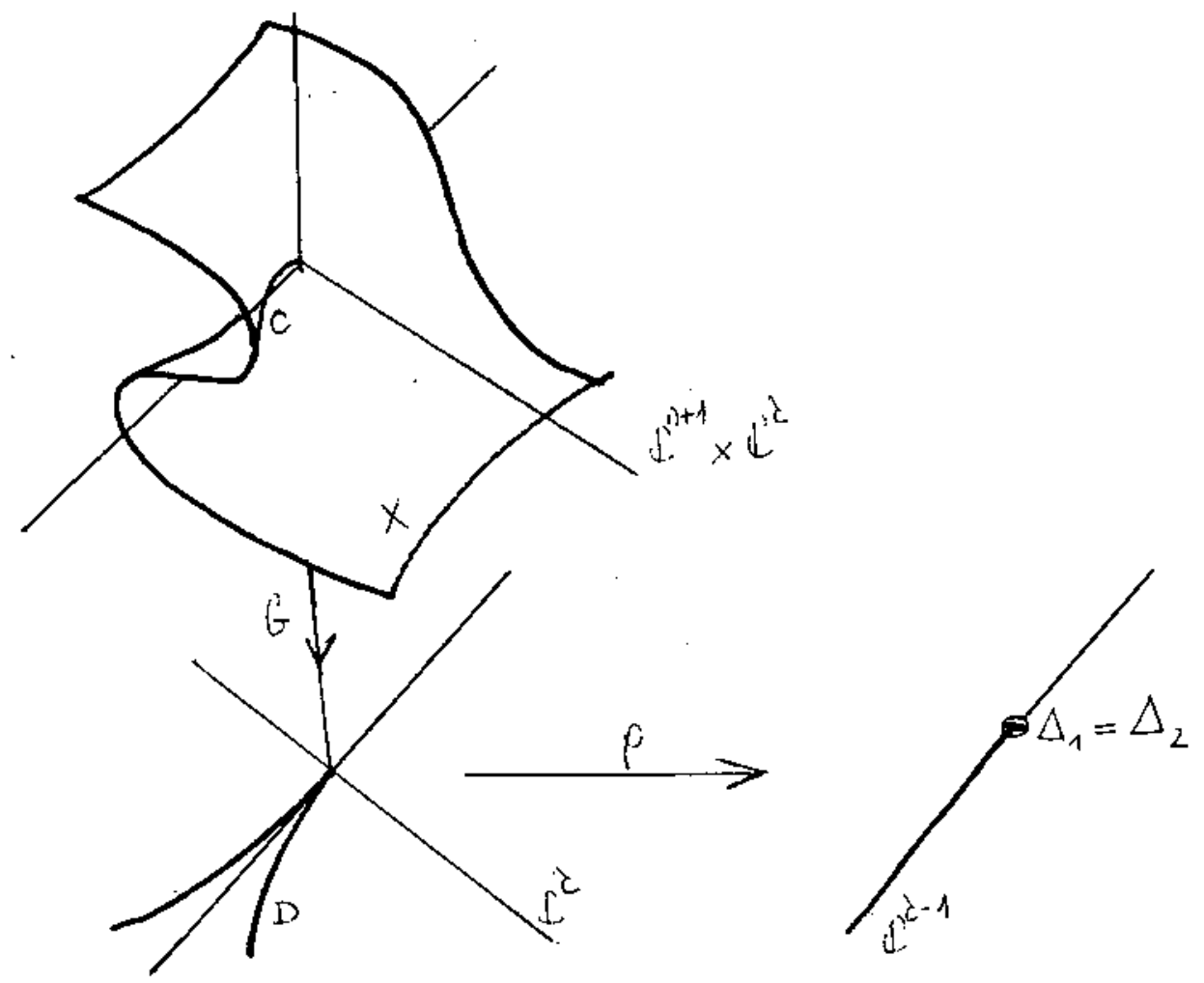

Figura 4

$y$ el espacio discriminante para $f=x^{4}$ es
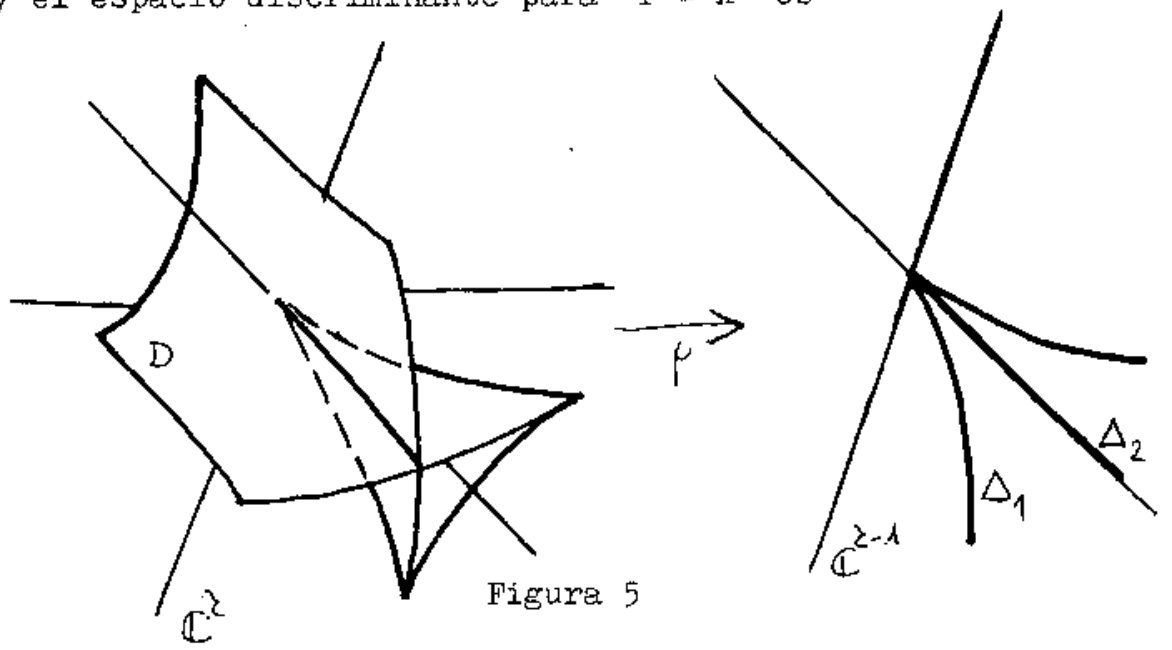
Si ahora tomenos un pinto $a=\left(a_{1}, \ldots, a_{\tau-1}\right) s \mathbb{d}^{t^{-1}}$ fuers de $\Delta$, la recta $Y_{a}=\left\{t_{1}=a_{1}, \ldots, t_{\tau-1}=a_{\tau-1}\right\} \quad c^{\tau} \mathbb{C}^{\tau}$, corta a $D$ en $\mu$ puntos distintos cuyo fibre presente un único punto singular no degenerato, asi, restringiendo $G$ it 19 imagen inversa de $\mathrm{Y}_{3}$, tencremos une aplicación

$$
f_{a}=G_{1}: G^{-1}\left(Y_{z_{2}}\right) \rightarrow Y_{a}
$$

con s6lo un número fjnito $\mu$ de puntos criticos no degererados, luego if $\mathrm{i}_{\mathrm{a}}$ es une fibración de Picard-Lefschetz! A una aplicación como ésta se le llama una morsificación de f. Aplicando el teorema de Ehresmann a la restricción de $G$ a $X-G_{T}^{-1}(D)$ con represertitantes adecuados se ve que

$$
G_{1}: X-G^{-1}(D) \rightarrow S-D
$$

es un fibrado $c^{\infty}$ localmente trivial, cuya restriceión a la recta $Y_{0}$ coincide con la fibración de Milnor, de forma que le fibra típica del fibrado $G_{1}$ es también la fibra de Milnor, y cuya restricción a una recta genérica $Y_{a}$ es una fibración de Pictird-Iefschetz: ésta es la imacen estereográfica que quería mostrar y que aglutina una enorme cuntidaa de información sobre la singularidad. Ver hasta que punto esta informecibn, en principio analítica, lo es también topológica es un interesante problema que está por resolver.

Voy a mostrar cbmo se puede utilizar la deformación semi-universal pare el cálculo de la morodromía algebraica de $\left(\mathrm{x}_{0}, \mathrm{x}_{0}\right)$. Para ello tomanos en $\mathrm{Y}_{0}^{*}=\mathrm{Y}_{0}-\{0\}$ un punto base $z_{0}$, de forma que la monodronía esté inducida por el camino $w:[0,1] \rightarrow S^{1}, w(t)=e^{2 \pi i t}$, entonces se puede escoger un ac ${\mathbb{c ^ { 2 }}}^{\tau-1}-\Delta$ de forma que todos los velores críti$\cos b_{\uparrow}, \ldots, b_{\mu}$ de $f_{a}$ estén en $Y_{a}$ dentro del disco de radio $\left|z_{o}\right|$, si ahora escogemos un camino $\gamma$ en $\mathbb{C}^{\top}-D$ que una $z_{0}$ con el origen de $\mathrm{Y}_{a}$, y caninos elementales $\mathrm{v}_{1}, \ldots, \mathrm{v}_{\mu}$ en $Y_{a}$ que no rodeen más valor crítico que $b_{1}, \ldots, b_{11}$, resp., $y$ que no se corten dos a dos, resultará que w es homotopo en 
$\mathbb{C}^{\tau}-\mathrm{D}$ a $\gamma^{-1} \mathrm{y}_{\mu} \ldots \mathrm{v}_{1} \gamma$, de forma que $\mathrm{la}_{\mathrm{a}}$ morodromia $\mathrm{h}_{*}=\mathrm{h}_{\mathrm{W}}$ será equivalente a la monodromía $h_{\mu} \ldots h_{1}$ de forma que la monodromía Jocal de Milnor sobre $Y_{0}$ (un solo punto crítico degenerado) se obtjere conociendo la monodrcmía Elobal de Picard-Lefschetz sobre $Y_{a}$ ( $\mu$ puntos críticos no degererados).

Ejemplo: Siguiendo con los puntos cuspidales, tomeros $\mathrm{x}^{3}+\mathrm{y}^{2}=0$ y veamos cómo se obtiene su monodramía algebraica utilizando la monodromía global. de P-I. Si en $\mathbb{C}^{2}(\mu=2)$ tomo solo le parte real de $t_{1}$, podré representar en $\mathrm{R}^{3}$ la situación

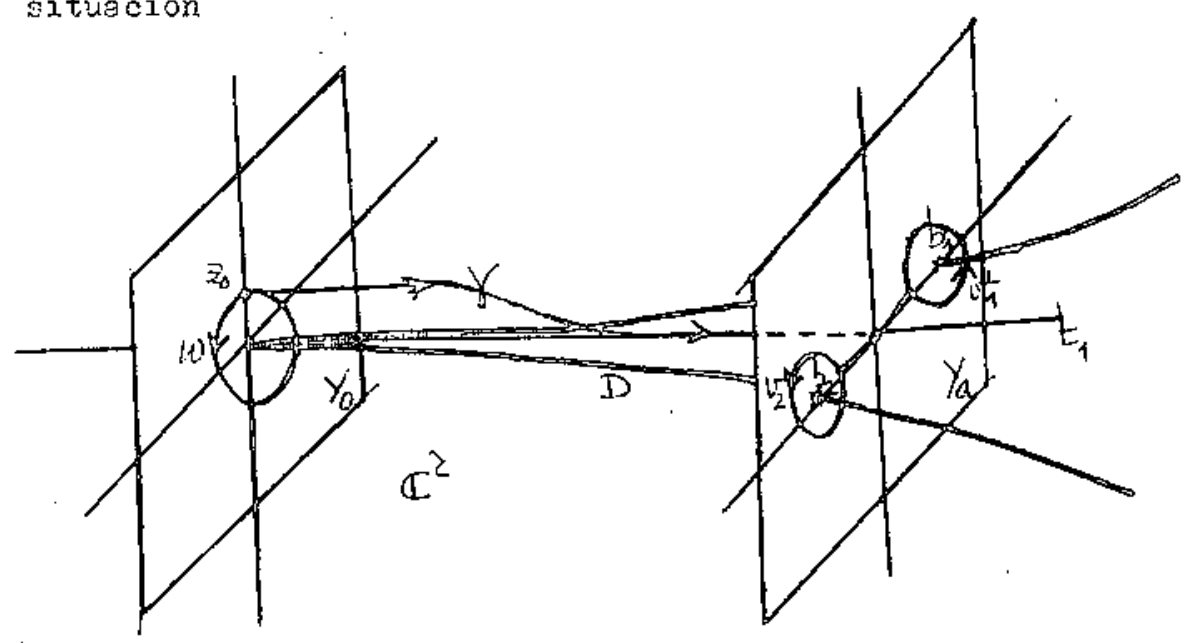

Figura 6

tomenos como base de $H_{1}(F, z)$ los ciclos evanescentes $s_{1}, s_{2}$ correspondientes a $\beta_{1}, \beta_{2}$, (esto es siempre posible por un teorema de [9]), en este caso es claro que

$$
\left\langle s_{1}, s_{1}\right\rangle=\left\langle s_{2}, s_{2}\right\rangle=0 \quad y \quad\left\langle s_{1}, s_{2}\right\rangle=\left\langle s_{2}, s_{1}\right\rangle=1
$$

de forma que aplicando las f6rmulas de P-I resulta

$$
n_{1}=\left[\begin{array}{ll}
1 & 1 \\
0 & 1
\end{array}\right] \quad y \quad n_{2}=\left[\begin{array}{cc}
1 & 0 \\
-1 & 1
\end{array}\right]
$$

$y$ en definitiva nos da: 


$$
h=h_{2} h_{1}=\left[\begin{array}{cc}
1 & 1 \\
-1 & 0
\end{array}\right]
$$

\section{RQUTVALENCTA ANAITTI.CA}

Hasta shora he mostrado un método de clasificación esencialmeste topológico cuyo origen se encuentra en R. Thom y su teoría de catástrofes. En efecto, aunque la teoría de cetástrofes está inicialmente formulada pars. gérmenes de funciones reales y utiliza la equivolencia diferenciable, los hechos básicos son que allí las singularidades se clesifican por su codimersión (equivalente a p-1 en el caso complejo) y que la clasificación que se. obtiere para codimensión $\leqslant 4$ es finita: las siete catástrofes elementales ya famosas. Sin embargo, exístía un bien conocido ejemplo de H. Whitney, precisamente las cuatro rectas que he citado er el apertado 1, que mostraba la no finitud del número de clases no equivalentes de gérmenes de codimensión 8 (ver también [39]). Fue así como R. Thom propuso sustjtuir la equivalencia diferenciatle por lat basada en el tipo topológico, a fin de mantener la clasificación por codimensiones y conjeturando, acertadimente como henos visto en 2, que el número de clases de férmenès de tipo topológico distinto pero de une misma codimensión sería entonces finito.

No obstante, este método ha sjỏo contestado por V.I. Arnold, quien ha propuesto otro sistema de clasificación como alternativa. Ia propuesta de Amold consiste en no abandonar la equivalencia diferenciable, o equivalencia analítica, en el caso complejo, pero sí abandonar la clasificación basada en la codimensión! Concretamente lo que propone aroold es un nuevo invariante analítico: la modalidad del germen, y una clasificación según esta modalidad, llegando a decir:

"... algebraicilly the most natural classifics- 
tion theoren are not those of singularities of small codimension c or of small multiplicity $\mu$, but the classification of singularities with stall modules number, $\mathrm{m}^{\text {" }}$ [3]

Esclarecer Ia relacion existente entre esta clam sificación y la topológica (vex tacla adjunta), y, en particular, explicar qué conexión existe entre las series de Arnold y los estratos de Fukuda. la modalidad y la dimersión del estrato (ver [8]),... sería, a mi entender, un decisivo avance en la clasjificación de sirgularidados.

Sin pretender que to que sigue sea una introduccion en el tena (ver, por ej., en [2] la bibijografía del propio Arnold) sí quievo comentar un resultado suficientemente arumático.

Antes explicaré cóno define Arnold la modalidad: si $f:\left(\mathbb{C}^{\mathrm{n}}, 0\right) \rightarrow(\mathbb{0}, 0)$ es un gernen de función holomorfa cun punto oritico aislado el origen, y suponenos que f esté dada por un polinomio de grato $k$ (ver [32]), entonces $f$ define una clase $\tilde{f}$ en el espac $=$ vectorial de dimensión finita $\underline{m}^{2} / \underline{m}^{k+1}$, siendo in el ideal maximal de las funciones nulas en el origen. Ahora bien, sobre este espacio $\underline{m}^{2} / \underline{m}^{k+1}$ actúa el grupo $\mathrm{G}$ de los automorfismos analíticos $\left(\mathbb{E}^{\mathrm{n}}, 0\right) \rightarrow\left(\mathbb{e}^{\mathrm{n}}, 0\right)$, y se Ilama modalidad de $\mathrm{f}$ al mínimo número 
CLASIFICACION TOPOLOGICA DE LAS SJNGULARIDADES DE NUMERO DE MILNCR $\leqslant 10$ (ver [3], [17], [29], [45]).

\begin{tabular}{|c|c|c|c|c|c|c|c|c|c|c|}
\hline$\mu$ & 1 & 2 & 3 & 4 & 5 & 6 & 7 & 8 & 9 & 10 \\
\hline \multirow{3}{*}{0} & $A_{1}$ & $A_{2}$ & $A_{3}$ & $\mathrm{~A}_{4}$ & $A_{5}$ & $\mathrm{~A}_{6}$ & $A_{7}$ & $A_{8}$ & $A_{9}$ & $A_{10}$ \\
\hline & & & & $D_{4}$ & $D_{5}$ & $\mathrm{D}_{6}$ & $D_{7}$ & $D_{8}$ & $D_{9}$ & $\mathrm{D}_{10}$ \\
\hline & & & & & & $E_{6}$ & $\Phi_{7}$ & $E_{8}$ & & \\
\hline \multirow{5}{*}{1} & & & & & & & & & $T_{3,3,4}$ & $\mathrm{~T}_{3,4,4}$ \\
\hline & & & & & & & & & & $\mathrm{T}_{2,4,5}$ \\
\hline & & & & & & & & & & ${ }^{\mathrm{T}} 2,3,6$ \\
\hline & & & & & & & & $P_{8}$ & $x_{9}$ & $J_{10}$ \\
\hline & & & & & & & & & & $Q_{10}$ \\
\hline
\end{tabular}

sierdo, salvo adición de una forma cuedrática no degererada en las restantes variables,

$$
\begin{aligned}
A_{m}: & x^{m+1}, \quad m \geqslant 1 \\
D_{m}: & x^{m-1}+x y^{2}, \quad y \geqslant 4 \\
E_{6}: & x^{4}+y^{3} \\
E_{7}: & x^{3} y^{3} y^{3} \\
E_{8}: & x^{5}+y^{3} \\
T_{P_{9},}: & x^{p}+y^{4}+z^{r}+a x y z, \quad a \neq 0 \\
P_{8}: & x^{3}+y^{3}+z^{3}+a x y z, \quad a^{3}+27 \neq 0 \\
Z_{9}: & x^{4}+y^{4}+a x^{2} y^{2}, \quad a^{2} \neq 4 \\
J_{10}: & x^{3}+y^{6}+a x^{2} y^{2}, \quad 4 a^{3}+27 \neq 0 \\
Q_{10}: & x^{3}+y^{4}+y z^{2}+a x y^{3}
\end{aligned}
$$

II para el que existe un entorno de $\tilde{f}$ recubierto por un número finito de familias mónarátricas de órbitas jel grupo G. A los gérmenes de modalided cero se les llama simples. 
Con este prólogo el resiltado de Arnold dice: Teorema $11(\operatorname{ver}[3]): f:\left(\mathbb{d}^{n}, 0\right) \rightarrow(\mathfrak{c}, 0)$ es un germien de función simple si, y sólo si, f es ana?íticamente equivalente a uno de los siguientes:

$$
\begin{aligned}
& A_{m}: \quad x_{1}^{m+1}+x_{2}^{2}+x_{3}^{2}+\ldots+x_{n}^{2}, m \geqslant 1 \\
& D_{m}: \quad x_{1}^{m-1}+x_{1} x_{2}^{2}+x_{3}^{2}+\ldots+x_{n}^{2}, \quad m \geqslant 4 \\
& E_{6}: \quad x_{1}^{4}+x_{2}^{3}+x_{3}^{2}+\ldots+x_{n}^{2} \\
& E_{7}: \quad x_{1}^{3} x_{2}+x_{2}^{3}+x_{3}^{2}+\ldots+x_{n}^{2} \\
& E_{3}: \quad x_{1}^{5}+x_{2}^{3}+x_{3}^{2}+\ldots+x_{n}^{2}
\end{aligned}
$$

Aunque a partir de aquí se obtienen interesantes caracterizaciones de los gémenes simples utilizando la monothromía (citemos como ejemplo: f es simple sji el grupo de monołromía de la deformación semiuniversal de $f$ es finito) lo más destacable es que estas singularidades simples han ido resurgiendo en distintos estudios desde hace más de cien años. No afectar la condición de adjunción [13], racionalidad [4], elipticidad [43],... han sido criterios de clasificación que han aparecido a lo largo de estos cien años y que han conducido siempre a las mismas singularidades simples.

Explicaré concisamente la forma más antigua de obtención de estas singularidades, es debida a Schwartz y F. Klein, y aparece ligada al estudio de las funciones automorfas poliedrales. Brevemente recordaré que dichas furciones se obtienen como inversas de las funciones multiformes que se obtienen por prolongación analítica a partir de las transformaciones conformes schwartzianas, que aplican el semiplano superior del plano cumplejo sobre un triángulo curvilíneo. Según sea la sura de los ángulos de dicho trián gulo mayor, igual a menor que $\pi$ se obtiene como imagen de la función multiforme la esfera (caso elíptico), el plano 
complejo (caso parabólico) o el seniplano hiparbólico de Poincaré (caso hiperbólico), respectivamente.

En el caso elíptico, único que quiero seguir, se obtiene ung' pavimentación de la esferi de Riemann formada por los triángulos curvilíneos engendrados por reflexiones sucesivas a partir del triangulo curvilíneo original. Resul ta ahora que esta pavimentación se mantiene invariante por un grupo finito $\Gamma$ ' de transformaciones de köbius, $\Gamma^{\prime} c \operatorname{PSL}(2, c)$, así las funciones inversas que estamos buscondo son funciones automorfas respecto a $\Gamma$ '. Pero como las únicas funciones meromorfas sobre la esfera son las funciones racionales, tenemos que, tomando coordenadas. proyectivas, 10 que buscinos son cocientes de polinomios homogéneos en dos variables del mismo grado e invariantes por $\boldsymbol{\Gamma}$, antiimagen de $\Gamma^{\prime}$ en $\mathrm{SL}(2, \mathbb{C})$. Así para resolver el problema inicial bastará con que encontremos el álgebra, $\mathrm{A}^{r}$, de polinomios homogéneos en dos variables invariantes por $r$, y aquí está la sorpresa:

Teorema 12 (Schwartz-Klein, ver [14], [25]): Si $P$ es un gripo finito de $\mathrm{SL}(2, \mathbb{C}), \mathrm{A}^{r}$ está engendrada por tres polinomios homogéneos $\mathrm{x}, \mathrm{y}, \mathrm{z}$ que satisfacen entre ellos la relación (syzygy):

donde

$$
f(x, y)+z^{2}=0
$$

$$
\begin{aligned}
& f(x, y)=x^{m}+y^{2}, \text { si } \Gamma \text { es el griapo cíclico de orden m. } \\
& f(x, y)=x^{m-1}+x y^{2}, \\
& \text { si } \Gamma \text { es el grupo diedral binario } \\
& \text { de orden } 4 m-4 .
\end{aligned}
$$

$f(x, y)=x^{4}+y^{3}$, si $\Gamma$ es el grupo tetraedral binario. $f(x, y)=x^{3} y+y^{3}$, si $\Gamma$ es el grupo octaedral binario. $f^{\prime}(x, y)=x^{5}+y^{3}$, si $\Gamma$ es el grupo icosaedral binario. jexactamente las singularidades simples en $\mathbb{c}^{2}$ ! 
Ya sólo me queda agradecér a J. Ferrer, F. Panyella y F. Puejta la ayuda que me prestaron en la elaboración de este trabajo, ni reconociniento a los tres.

\section{BIBLIOGRAFIA}

[1] A'Campo, N.: Le nombre de Lefschetz d'une monodromie, Indaf. Math., 35 (1973), 113-118.

[2] Arnold, V.I.: Critical points of smooth functions, Proc. Int. Congress Math. (1974), vol. $1,19-39$.

[3] Arnold, V.I.: Local normal forms of functions, Invent. Math. 35 (1976), 87-109.

[4] Artin, M.: On isolated rational singularities of surfaces, Amer. J. Math., 88 (1966), 129-136.

[5] Brauner, K.: Zur geometrie der funktionen zweier komplexen veranderlichen III, IV, Abh. Math. Sem. Hamburg, .6 (1928), 8-54.

[6] Brieskorn, E.: Examples of singular normal complex spaces which are topological manifolds, Proc. Nat. Acad. Sci. USA, 55 (1966), 1395-1397.

[ 7] Brieskorn, E.: Beispiele zur differentialtopologie von singularitäten, Invent. Math. 2 (1966), 1-14.

[8] Brieskorn, E.: Rationale singularitäten komplexer flachen, Invent. Wath. 4 (1967-68), $336-358$.

[ 9] Brieskorn, E.: Die monodromie der isolienten singularitäten von hyperflachen, Nianscripta Math. 2 (1970), 103-161.

[10] Burau, W.: Kennzeichnung der schlauchknoten, Abh. Math. Sem. Hamburg, 9 (1932), 125-133. 
[11] Buxalx, W.: Kennzeichnung der schlaucisverbettungen, Abh. Math. Sem. Hamburg, 10, (1934),285-397.

[12] Durfee, A.: Fibered knots and algebraic singularities, Topology 13, (1974), 47-60.

[13] Ir Val, P.: On-isolated singularities which do not affect the conditions of adjunction, I, II, III, Proc. Cambridge Philos. Soc. 30, (1934), 453-465, 483-491.

[14] Du Val, P.: Homographies, quaternions and rotations, Oxford, 1964.

[15] Fary, I.: Cohomologie des varietés algébriques, Annals of Nath. 65 (1957), 21-73.

[16] Fukuda, T.: Types topologiques des polynônes, Publ. . Math. I.H.E.S. 46 (1976), 87-106.

[17] Gabrielov, A.M.: Dynkin diagrams for unimodal singularities, Funct. Anal. 8 (1974), 192-196.

[18] Gabrielov, A.M.: Bifurcations, Dynkin diagrams and modality of isolated singularities, Funct. Anal. Appl. 8 (1974), 94-98.

[19] Grauert, H.: Uber die deformation isolierter singularitäten analytischer mengen, Invent. . Nath. 15 (1972), 171-198.

[20] Hirzebruch, F.: The topology of normal singularities of an algebraic surface (d'aprés Mumford), séminaire N. Bourbaki 15, (1962/6.3), n. 250.

[21] Hirzebruch, F.: Singularities and exotic spheres, Séminaire N. Bourbaki, 19 (1966/67), n. 314 .

[22] Hirzebruch, F. y Mayer, K.H.: Differenciarbe O(n)Mannigfal.tigbeiten, exotische sphären, und singularitäten, Lecture Notes 57 , Sprìnger-Verlag, 1968. 
[23] Kähler, K.: Uber die verzweigung einer algebraischen funktion sweier veranderlichen zu der umgebung einea singularen stelle, Math. zeit., 30 (1929), 188-204.

[24] King, H.C.: Topological type of isolated critical points, Annals of Math. 107 (1978), 385-397.

[25] Klein, F.: Lectures on the icosahedron and the solution of equations of the fifth degree, Dover, 1956.

[26] Kuiper, N.H.: Algebraic equations for nonsmoothable 8-manifolds, Fubl. Math. I.H.E.S. 33, (1967), 139-155.

[27] Lamotke, K.: Die homologie isolierter singularitäten, Math. Z. 143 (1975), 27-44.

[28] Lê, D.T.: Calcul des eycles évanouissants des hipersurfaces complexes, Ann. Inst. Fourier, 4 (1973), 261-270.

[29] Lé, D.T. y Ramanujan, C.P.: The invariance of Milnor's number implies the' invariance of the topological type, Amer. T. Math. 98 (1976), 67-78.

[30] Lefschetz, S.: I'analysis situs et la géométrie algebrique, Gauthiers-Viliars, 1924.

[31] Levine, J.: An algebraic classification of some knots of codimension two, Comment. Math. Helvet. 45 (1970), 185-198.

[32] Mather, J.: Stability of $\mathrm{C}^{\Phi}$-mappings III, finitely determined map-germs, Publ. Math. I.H.E.S. 35 (1968), 127-156.

[33] Milnor, J.: Singular points of complex hipersurfaces, Annals of Math. Studies 61, Princeton Univ. Press, 1968. 
[34] Mumford, D.: The topology of normal singularities of an algebraic surface and a criterion for simplicity, Publ. Math. T.H.E.S. 9 (1961), 229-246.

[35] Pham, F.: Formules de Picard-Lefschetz généralisées et ranification des intégrals, Bull. Soc. Math. France 93 (1965), 333-367.

[36] Pham, F.: Introduction a l'xude topologique des singujarités de Landau, Gauthier-Villars, 1967.

[37] Picard, E. y Simart, G.: Théorie des fonctions algébriques de deux variables indépendants, vol I, Gauthier-Viliars, 1897.

[38] Sebastiani, M. y Thom, R.: Un résultat sur la monodromie, Invent. Math. 13 (1971), 90-96.

[39] Siersma, D.: The singularities of $c^{\infty}$-functions of right-codimension smaller or equal than eight, Indag. Math. 35 (1973), 31-37.

[40] Teissier, B.: Cycles evanescentes, sections planes et conditions de Whitney, Astérisque $7 / 8$ (1973), 285-361.

[41] Thom, R.: La stabilité topologique des applications polynomials, L'Enseignement Math. 8 (1962), 24-33.

[42] Thom, R.: Stabilité structurelle et morphogénese, Benjamin, 1972.

[43] Tjurina, G.N.: The topological properties of isolated singularities of complex spaces of codimension one, Math. USSR - Izvestija, 2 (1968), 557-571:

[44] Tjurina, G.N.: Locally semiuniversal flat deformations of isolated singularities of complex spaces, Math. USSR-Izvestija, 3 (1970), 957-999. 
[45] Varchenko, A.N.: Zeta-function of monodrony and Newton's diagram, Invent. Math. 37 (1976), 253-262.

[46] Zariski, 0.: Some open cuestions in the theory of singularities, Bull. A.M.S. 77 (1971), 481-491.

Departamento de Matemáticas, E.T.S.I.I.B. Universidad Politécnica de Barcelona. 